\title{
Flow Rate along the Length of the Swirling Vortex Axis at an Intake
}

\author{
Kerem TAŞTAN ${ }^{1}$ \\ Nevzat YILDIRIM ${ }^{2}$
}

\begin{abstract}
In this study, the characteristics of the flow in the region of swirling vortex are examined. The potential flow model based on the summing infinite number of spherical sinks along the vortex core is introduced to predict the flow field and the flow rate along the vortex axis. The flow towards the swirling vortex core has considerable effects on the radial velocity distribution within the ambient fluid flow region near the intake. The agreement between available test data relating to the radial velocity and the method introduced in this study is found to be satisfactory.
\end{abstract}

Keywords: Intake, submergence, swirling flow, vortex.

\section{INTRODUCTION}

Hydraulic problems attributed to the swirling vortex flow occurring at intakes are frequently encountered in practice (such as discharge reduction, loss of efficiency in pumps and turbines, vibration, air-entrainment, erosion in water-conveying structures etc). The previous studies on this issue may briefly be summarized in three categories that are as follows. i) Studies considering the critical submergence: Fully developed air-entraining vortices occur at intakes that have no sufficient submergence, $S$ (the vertical distance of the intake center to the fluid surface). There are several studies about the critical submergence $\left[S=S_{c}\right.$ at which the lower end (tip) of the air-core vortex just reaches the intake] include those by Denny [1], Anwar et al. [2], Hecker [3], Taştan and Yıldırım [4], Kocabaş and Yıldırım [5], Yang et al. [6], Yıldırım et al. [7], Sun and Liu [8]. ii) Studies relating to the modelling of main characteristics of air-core vortices are: Odgaard [9]; Suerich-Gulick et al. [10]; and Vatistas et al. [11]. iii) Studies relating to profile of the air-core vortex: Typical example works on this issue are by Anwar [12]; Vatistas et al. [13]; and Sun and Liu [8].

\footnotetext{
Note:

- This paper has been received on July 18, 2019 and accepted for publication by the Editorial Board on January 7, 2020.

- Discussions on this paper will be accepted by September 30, 2021.

- https://doi.org/10.18400/tekderg.593595

1 Gazi University, Department of Civil Engineering, Ankara, Turkey ktastan@gazi.edu.tr - https://orcid.org/0000-0003-1747-2496

2 Çankaya University, Department of Civil Engineering, Ankara, Turkey nevzaty@cankaya.edu.tr - https://orcid.org/0000-0002-0985-8084
} 
So far, in the available literature mentioned earlier, the following two classical points are considered for the potential flow region of the vortex. i) The circulatory velocity vectors are tangential to completely closed co-centric circular streamlines in a horizontal plane and varying in magnitude inversely with the radial distance from the vertical axis of the vortex (the center of the circular streamlines). The pressure distribution is hydrostatic. The entire ambient circulatory flow is in the form of an infinite number of concurrently rotating co-axial vertical cylindrical sink surfaces extending from the free surface to the bottom boundary and having the same vertical axis as the air-entraining vortex. ii) The path of each particle is a completely closed circular streamline with no swirling, and the entire profile of the air-core vortex is of a single closed-ended "hyperbolic paraboloid of revolution of circular crosssection" with constant flux of magnitude of vorticity (the net circulation is identical at all levels along the entire length of the air-core vortex).

The flow visualization in the study by Kocabaş and Yıldırım [5] indicated that the path lines of the particles are in the form of descending and converging unclosed lines that appear as a swirling-vortex filament. This indicates that in reality, the streamlines are not completely closed circular lines in the horizontal plane and they also have curvatures in the vertical plane. Therefore, the pressure distribution is not exactly hydrostatic. The circulatory ambient fluid is not in the form of rotating co-centric cylindrical sink surfaces and the circulation along the pathway axis of the vortex is not an identical constant. The circulation along the axis of the vortex increases from its minimum magnitude at the free surface to its maximum magnitude at the intake.

The air-core vortex has to be attributed to the submerged swirling vortex flow developed under the surface extending from the free-surface (air-boundary) to the intake. The exact nature of the swirling vortex flow developing under the water surface is still unknown. Especially the discharge towards any point along the axis of the swirling vortex flow-filament is needed to be known in order to predict the variation of the magnitude of the vorticity or circulation (along the axis of the swirling vortex) that is the main reason for the occurrence of the swirling vortex or air-core vortex. The available studies have not given any information or explanations relating to the flow rate along the axis of swirling vortex or air-core vortex. Taştan and Yıldırım [14] have found that the vortex core consists of hydraulically developed coaxial subsurface depressions (SSDs) towards which the flows are of coaxial spherical sink surface sectors (SSSSs) with centers changing along the axis of the vortex core towards the intake. They have presented an analytical analysis for the profile of the air-core vortex and SSSSs, and verified it by using available test data relating to the air-core vortex. However, they have not considered the flow rate along the axis of the vortex core. Note that, if an SSSS is in the form of a hemisphere then the SSSS is denoted by HSSS (hemispherical sink surfaces) in the present study.

This paper continues the work presented in the study by Taştan and Yıldırım [14]. The main difference is that the present study introduces a potential flow model based on the summing infinite number of SSSSs along the swirling vortex core (not necessarily the air-core vortex) to predict the flow field and the flow rate along the axis of the swirling vortex occurring at an intake. The flow towards the SSDs relating to the swirling vortex-filament has considerable effects on the radial velocity distribution within the ambient fluid flow region near the intake. The agreement between available test data and the method introduced in this study is found to be satisfactory. 


\section{THEORETICAL CONSIDERATION}

Whenever there exists an intake in an ambient fluid with net circulation; an unavoidable swirling vortex flow filament develops within the ambient fluid at the intake (Fig. 1a).

(a)

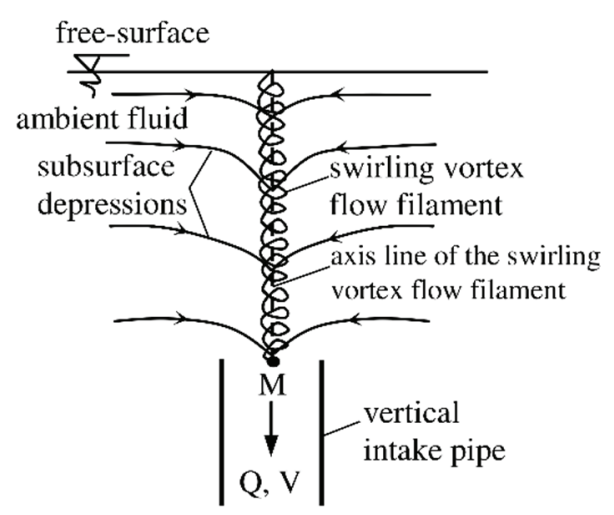

(b)

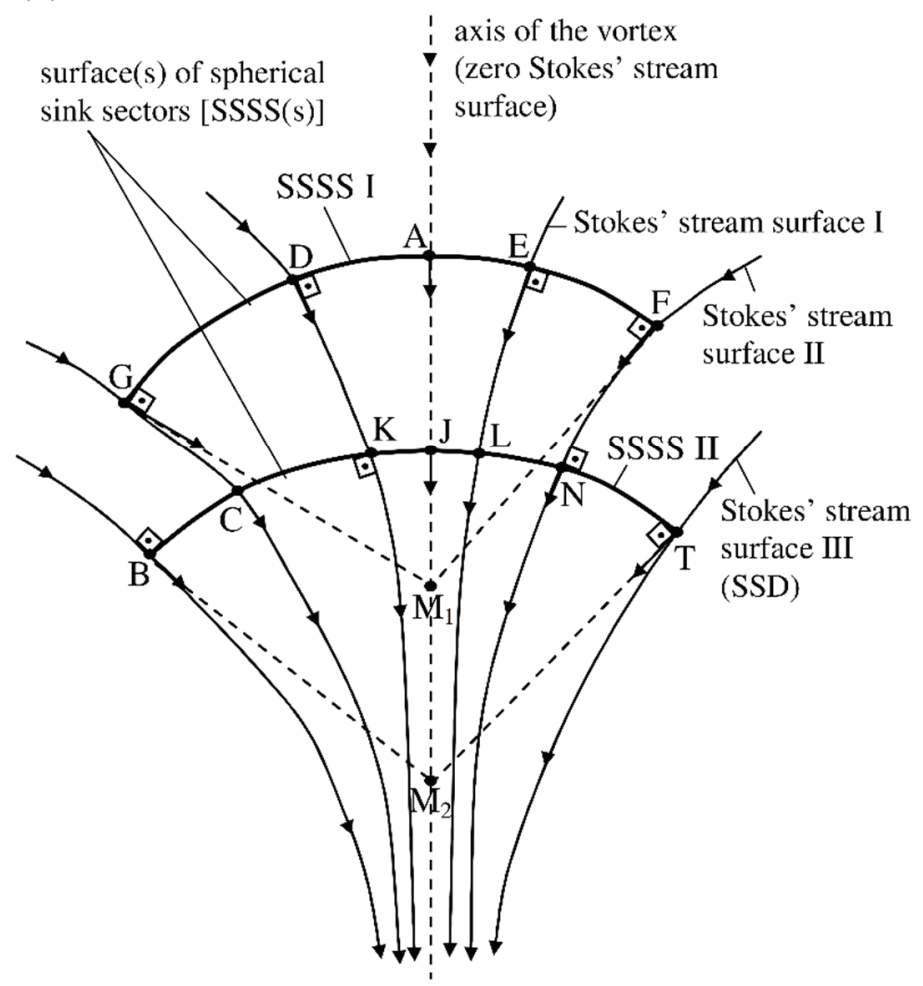

Figure 1- (a) SSDs and the swirling vortex filament,

(b) $\operatorname{SSSS}(s)$, and Stokes' stream surfaces or $\operatorname{SSD}(s)$ [14] 
The swirling motion of the fluid particles or swirling characteristic of the vortex-path line is due to the circulation attributed to that may be either self-developed due to the Coriolis effect or disturbances and rotations present within the ambient fluid flow towards the intake or induced, imposed, or loaded circulation on the ambient fluid flow field. Circulation is the essential and main reason for the development of a swirling vortex flow or air-core vortex in an ambient fluid with an intake. If there is no net circulation, a swirling vortex flow cannot develop. In the study by Taştan and Yıldırım [14] relating to the air-core vortex at an intake, it is proven that regardless of the flow and geometrical conditions the fluid flow towards the swirling vortex consists of hydraulically developed coaxial SSSSs of varying centers along the axis of hydraulically developed coaxial SSDs of point (spherical) sink character for the ambient fluid (Fig. 1b). The SSDs are a kind of depression intakes (DIs) whose boundaries are of Stokes' stream surfaces. In reality, all SSDs or DIs are open-ended through which the ambient fluid flow filling in the SSDs reaches and enters the intake.

In the study by Taştan and Yıldırım [14] it is stated that :

"The geometrical location of whole fluid particles that have an identical magnitude of radial velocity towards any chosen center point on the axis of the vortex depression (vortex path line) is an SSSS. Note that these particles belong to an infinite number of coaxial Stokes' stream surfaces (Figs. 1b and 2). For example, in Fig. 1b, the particles G, D, A, E, and F of different Stokes' stream surfaces [DI(s)] are on the same SSSS I and have identical radial velocity towards the center $\mathrm{M}_{1}$ of the SSSS I. Similarly, the particles B, C, K, J, L, N, and T of different Stokes' stream surfaces [DI(s)] are on the same SSSS II and have identical radial velocity towards the center $\mathrm{M}_{2}$ of the SSSS II."

The potential flow model is based on the summation of infinite number of SSSSs along the length of the vortex axis. The potential lines representing radial velocity are perpendicular to the stream surfaces of SSSSs. For this purpose, firstly it is necessary to prove that for each SSD there exist HSSSs or SSSSs in general. This is achieved in the following way.

Considering an air-core vortex depression and the coordinate system as indicated in Fig. 2 (basing on the formula developed by Vatistas et al. [13]; Sun and Liu, [8]) for the profile of an air-core vortex, Taştan and Yıldırım [14] have written as

$$
\frac{H_{r}-H_{0}}{h}=\frac{2}{\pi} \arctan \left(\beta R^{2}\right)
$$

where $R=r / r_{m}=$ dimensionless horizontal radial distance from the vertical centerline of the depression (vortex) or intake entrance; $r=$ horizontal radial distance from the vertical centerline of the air-core vortex depression (or intake entrance); $r_{m}=$ air-core vortex depression core radius (outer limit radius of the viscous region); $H_{r}=$ water surface elevation at $R ; H_{0}=$ elevation of the lower end or tip of the depression (air-core vortex); $h=$ total depth of the depression (air-core vortex); and $\beta=$ a coefficient relating to the flow conditions. In reality, the lower end (tip) of the air-core vortex depression should have a tiny opening but the surface tension (capillary) forces overcome the small inertia and gravity forces at the lower end of the air-core vortex. Therefore the lower end (tip) of the air-core vortex depression is seen as closed and slightly curved [14]. 


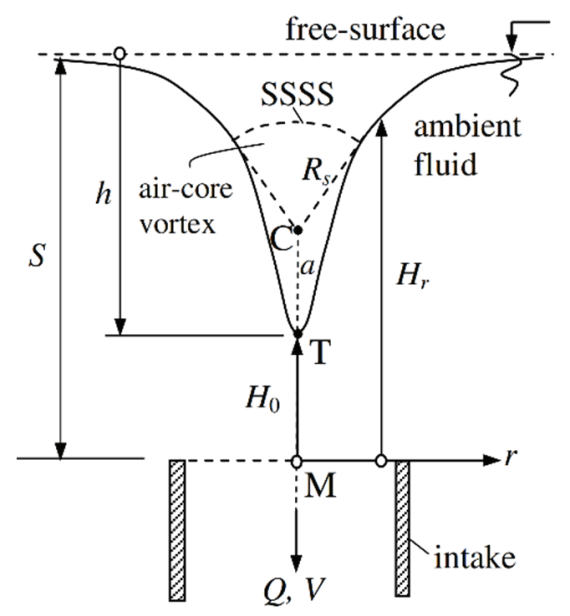

(a)

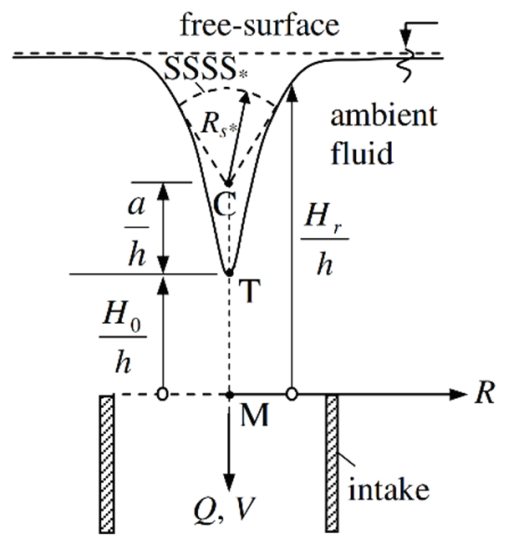

(b)

Figure 2 - Geometric variable definitions [14]

Consider an SSSS whose center C on the axis of symmetry of the intake entrance or air-core vortex is above the lower end (tip) of the air-core vortex for an amount of $a$ or $a / h$, as shown in Fig. $2 a$ and $b$. The SSSS can be described by the equation of a circle in which the horizontal dimension is $R$ and the vertical dimension is the radius of the circle expressed in terms of the geometry of the air-core vortex. For the circular arch, one can write [14],

$R^{2}+\left(\frac{H_{r}-H_{0}}{h}-\frac{a}{h}\right)^{2}=R_{s^{*}}^{2}$

where $R_{S^{*}}$ is the dimensionless radius of a different kind of SSSS that is indicated as SSSS* (in the dimensionless coordinate system of $H_{r} / h$ and $R$ ) in Fig. $2 \mathrm{~b}$. 
Taştan and Yıldırım [14] have shown that SSSS* and air-core vortex depression profile normally intersect (for example at intersection point A in Fig. 1) for which one can write

$$
R^{2}=\left[\frac{1}{\pi\left(\frac{H_{r}-H_{0}}{h}-\frac{a}{h}\right)}+\sqrt{\frac{1}{\pi^{2}\left(\frac{H_{r}-H_{0}}{h}-\frac{a}{h}\right)^{2}}-0.25}\right] \frac{2}{\beta}
$$

Substituting Eq. (3) in Eq. (1) (for the intersection point of SSSS and air-core vortex profile) it yields

$$
\left(\frac{H_{r}-H_{0}}{h}\right)=\frac{2}{\pi} \arctan \left\{2\left[\frac{1}{\pi\left(\frac{H_{r}-H_{0}}{h}-\frac{a}{h}\right)}+\sqrt{\frac{1}{\pi^{2}\left(\frac{H_{r}-H_{0}}{h}-\frac{a}{h}\right)^{2}}-0.25}\right]\right\}
$$

Note that Eq. (4) is independent of $\beta$.

To prove that there exist HSSSs or SSSSs for each SSD; consider any $\mathrm{i}^{\text {th }} \mathrm{SSD}$ (call it $\mathrm{SSD}_{\mathrm{i}}$ ) as indicated in Fig. 3. For simplicity let the origin of the coordinate system $\left(r, H_{r i}\right)$ or $\left(R_{i}\right.$, $\left.H_{r i} / h_{i}\right)$ is located at the lower end (tip) point $\mathrm{T}_{\mathrm{i}}$ of the $\mathrm{SSD}_{\mathrm{i}}$ (Fig. 3). Herein, $R_{i}=r / r_{m i} ; r_{m i}=$ core radius (outer limit radius of the viscous region) of the $\mathrm{SSD}_{\mathrm{i}} ; H_{r i}=$ elevation of the $\mathrm{SSD}_{\mathrm{i}}$ profile with respect to the lower end (tip) of the $\mathrm{SSD}_{\mathrm{i}}$ at $R_{i} ; h_{i}=$ the vertical distance of the top level of the $\mathrm{SSD}_{\mathrm{i}}$ at sufficiently large horizontal radial distance from the vertical central axis line of the $\mathrm{SSD}_{\mathrm{i}}$ or the intake centre. In reality an air-core vortex depression is also an SSD for which the depth of ambient fluid above it is not sufficient to fill out the SSD alone (SSD is filled out by both the ambient fluid and air as explained in the study by Taştan and Y1ldirım [14]. Therefore; Eq. (1) can be adopted for any $\mathrm{i}^{\text {th }} \mathrm{SSD}$ (call it $\mathrm{SSD}_{\mathrm{i}}$ ) provided that $H_{r i}, h_{i}, \beta_{i}$, and $R_{i}$ are used in lieu of their corresponding $\left(H_{r}-H_{0}\right), h, \beta$, and $R$ respectively as indicated in Fig. 3. For the profile of $\operatorname{SSD}_{\mathrm{i}}$ Eqs. (1) and (2) may be rewritten as;

$$
\begin{aligned}
& \frac{H_{r i}}{h_{i}}=\frac{2}{\pi} \arctan \left(\beta_{i} R_{i}^{2}\right) \\
& R_{i}^{2}+\left(\frac{H_{r i}}{h_{i}}-\frac{a_{i}}{h_{i}}\right)^{2}=R_{s i^{*}}{ }^{2}
\end{aligned}
$$

Herein, $R_{s i^{*}}=$ dimensionless radius of a different kind of $\operatorname{SSSS}_{\mathrm{i}}$ that is indicated as $\mathrm{SSSS}_{\mathrm{i}^{*}}$ (in the dimensionless coordinate system of $H_{r i} / h_{i}$ and $\left.R_{i}\right)$; and $a_{i}=$ vertical distance of the center point of the $\mathrm{SSSS}_{\mathrm{i}}$ to the lower end (tip) of the $\mathrm{SSD}_{\mathrm{i}}$ (Fig. 3b). Note that $H_{r i} ; h_{i} ; \beta_{i} ; R_{i}$; and $R_{S i}$ may change for all SSDs. Considering Eqs. (3) and (4); for the normal-intersection of the profiles of the $\mathrm{SSD}_{\mathrm{i}}$ and the $\mathrm{SSSS}_{\mathrm{i}}$ one may write 


$$
\begin{aligned}
& \left.R_{i}^{2}=\left[\frac{1}{\pi\left(\frac{H_{r i}}{h_{i}}-\frac{a_{i}}{h_{i}}\right)}+\sqrt{\frac{1}{\pi^{2}\left(\frac{H_{r i}}{h_{i}}-\frac{a_{i}}{h_{i}}\right)^{2}}-0.25}\right] \frac{2}{\beta_{i}}\right] \\
& \frac{H_{r i}}{h_{i}}=\frac{2}{\pi} \arctan \left\{2\left[\frac{1}{\pi\left(\frac{H_{r i}}{h_{i}}-\frac{a_{i}}{h_{i}}\right)}+\sqrt{\frac{1}{\pi^{2}\left(\frac{H_{r i}}{h_{i}}-\frac{a_{i}}{h_{i}}\right)^{2}}-0.25}\right]\right\}
\end{aligned}
$$

(a)

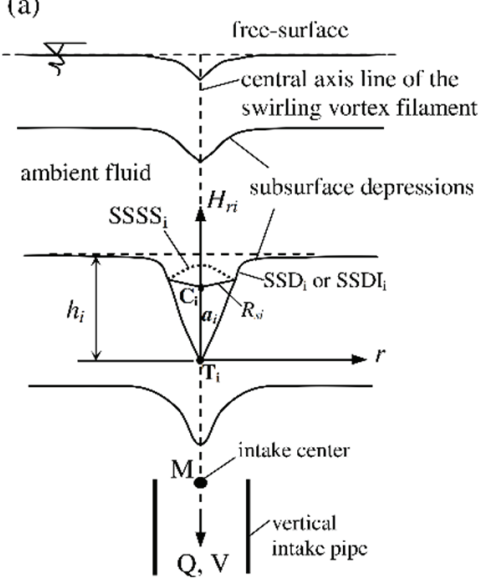

(b)

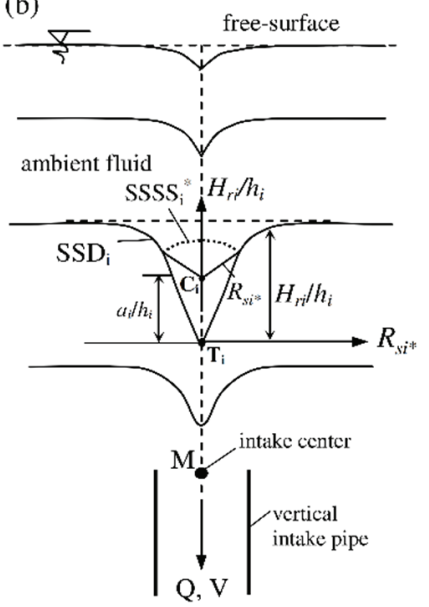

Figure 3 - SSD(s) and relating geometric parameters 
Eq. (8) can be solved for $H_{r i} / h_{i}$ by a trial and error procedure for a chosen magnitude of $a_{i}$ $/ h_{i}$ indicating that for every point on the axis of the vortex there corresponds an SSSS. It can be shown that Eq. (8) has only one real root within the domain of solution by constructing a graph of assumed values of $H_{r i} / h_{i}$ with respect to computed values of $H_{r i} / h_{i}$ for all possible $a_{i}$ 's.

For simplicity it may be advised to study the case of $a_{i}=0$ first. For $a_{i}=0$ the $\mathrm{SSSS}_{\mathrm{i}}$ has the same center as the lower end (tip) for which Eqs. (5)-(8) give

$H_{r i} / h_{i}=0.6033$; or $H_{r i}=0.6033 h_{i}$; and $R_{i}=0.8343$; or $r=0.8343 r_{m i}$

Now, one can go to Fig. 4a (with $a_{i}=0 ; H_{r i}=0.6033 h_{i}$; and $r=0.8343 r_{m i}$ that correspond the point B in Fig. $4 \mathrm{a}$ ) and write the equation for a circle (whose revolution gives an $\mathrm{SSS}_{\mathrm{i}}$ ) of radius of $R_{s i}$ as follows:

$$
\begin{aligned}
& r^{2}+H_{r i}^{2}=R_{s i}^{2} \\
& \left(0.8343 r_{m i}\right)^{2}+\left(0.6033 h_{i}\right)^{2}=R_{s i}^{2}=\text { constant } \\
& h_{i}^{2}=\text { constant }-1.912 r_{m i}^{2}
\end{aligned}
$$

(a)

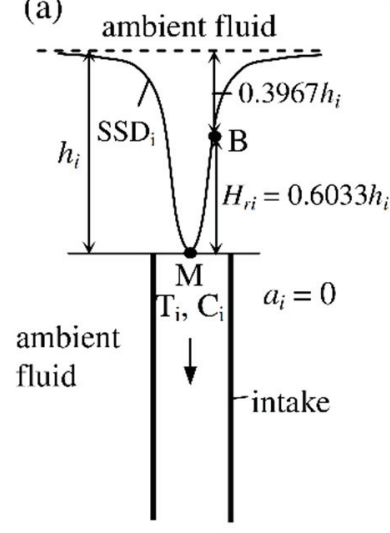

(b)

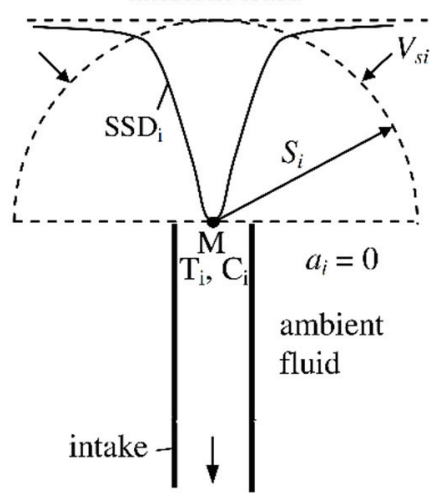

Figure $4-S S D_{i}$ for the case of $a_{i}=0$

Considering the air-core vortex depression, the test results of Sun and Liu [8] indicate that $r_{m}$ is a constant for the vertical distance from the top level of $\mathrm{SSD}_{\mathrm{i}}$ less than or equal to $0.7 S_{c}$ (or $\left.H_{r} / S_{c}=1-0.7=0.3\right)$.

Noting that the air-core vortex depression is also one of the SSDs; for an $\mathrm{SSD}_{\mathrm{i}}$ Eq. (9) indicates that for $a_{i}=0$, the vertical distance of the point B (Fig. 4a) from the top level of $\mathrm{SSD}_{\mathrm{i}}$ is $\left(h_{i}-H_{r i}\right)_{\text {at B }}$ or, $\left(h_{i}-0.6033 h_{i}\right)=0.3967 h_{i}<0.7 h_{i}$, this infers that $r_{m i}$ in Eq. (10c) can also be taken as a constant. Thus, Eq. (10c) can be rewritten as 
$h_{i}^{2}=\mathrm{a}$ constant

One can multiply both sides of Eq. (11) by $2 \pi$ and get

$2 \pi h_{i}^{2}=$ constant

Equation (12) indicates that regardless of flow and geometrical conditions (including the profile of the intake-entrance) there exists an $\mathrm{HSSS}_{\mathrm{i}}$ of radius of $h_{i}$ (on upstream side of the tip point $\mathrm{T}_{\mathrm{i}}$ ) that has the same centre as the point $\mathrm{T}_{\mathrm{i}}$, and with the base normal to the central axis of the swirling vortex filament at point $T_{i}$ as indicated in Fig. 4.

Considering Eq. (12), the discharge, $q_{i}$, towards the centre $\mathrm{T}_{\mathrm{i}}$ of the $\mathrm{HSSS}_{\mathrm{i}}$ can be written as

$$
q_{i}=\left(2 \pi h_{i}^{2}\right) V_{s i}
$$

where $V_{s i}=$ radial velocity at the $\mathrm{HSSS}_{\mathrm{i}}$ whose magnitude depends on the flow and geometrical conditions. $V_{s i}$ may be measured.

Equation (13) infers that the discharge towards any point under consideration on the centerline of the swirling vortex filament is equal to that through the $\mathrm{HSSS}_{\mathrm{i}}$ (on upstream side of the point $T_{i}$ ) that has the same center as $T_{i}$, and the base normal to the central axis of the swirling vortex filament at $\mathrm{T}_{\mathrm{i}}$ (Fig. 4). This discharge is valid for the flow on the upstream side of the point $T_{i}$. Note that the above conclusion is reached for $a_{i}=0$. If similar analysis is done for a given $a_{i} \neq 0$ the same conclusion is to be obtained. Existence of HSSSs within the flow field along the path of the swirling vortex does not depend on the value of $a_{i}$.

Physically the lower ends (tips) of whole SSDs at upstream of a point on the axis of the swirling vortex flow-filament must one after another reach (and pass from) that point. Because these SSDs have their own respective HSSSs, one can draw infinite number of concentric HSSSs with different radii at that point as indicated in Fig. 5. This means that at every point along the axis of the swirling vortex flow infinite number of concentric HSSSs having identical discharge can be drawn. Not to make the figure crowded HSSSs at only a few points are presented in Fig. 5.

As for the practice, Eq. (13) indicates that if $V_{s i}$ at a given radial distance of $h_{\mathrm{i}}$ to the point under consideration on the axis of the swirling vortex filament is measured, then the discharge $\left(q_{i}\right)$ towards that point (center of the HSSS) can be calculated. To measure the radial velocities, a point on the path of the swirling vortex should be chosen. Then a velocity meter (i.e., a pitot tube) should be radially aligned at the point that has required radial distance (radius of SSS) to the point under consideration.

By means of this method one can know the magnitude of the discharge participating in the vortex filament (vorticity feeding-discharge) at any level along the axis of the swirling vortex flow-filament.

Figure 5 indicates that HSSSs radially shrink towards their center. As HSSSs radially shrink they must simultaneously rotate [due to the forces caused by non-uniformities in the ambient flow media, the Coriolis force, and imposed or induced circulation] about their axis (axis of 
the SSDs). This may be the reason why the swirling vortex flow is observed to be a swirling filament (Fig. 1a).

Physically it is seen that HSSSs having centers on the central axis line of the swirling vortex filament at upstream of the intake center M (Fig. 5) have open bases through which the fluid flows or escapes (evacuation of the fluid within the HSSSs occurs). The base of the HSSS or SSSS having the same center and discharge as the intake has the opening of the intake entrance. Similar to the swirling vortex filament above the intake as indicated in Figs. 1-4, in reality another swirling vortex filament also exists below the intake that is not visible (not indicated in Figs. 1-4) since it is blocked by the vertically downward flowing intake pipe. Therefore, similar to the HSSSs above the intake level, one can also draw HSSSs below the intake level as indicated Fig. 5c. Note that spatial growth of the swirling vortex from freesurface to the intake is not considered in this study.

(a)

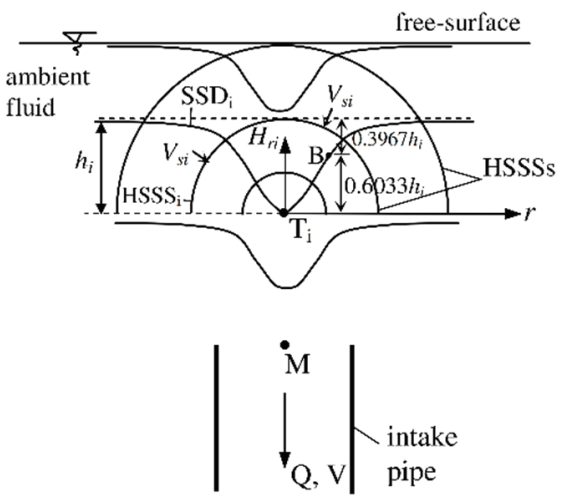

(b)

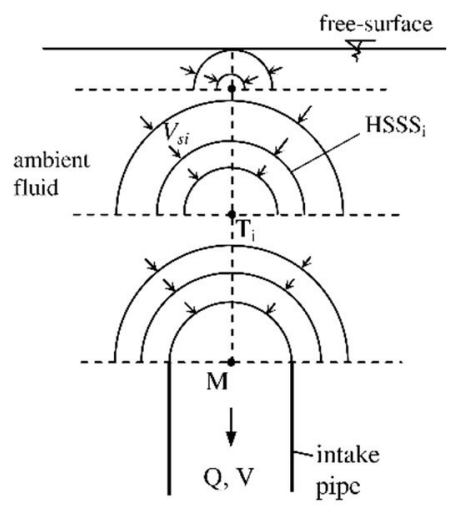

(c)

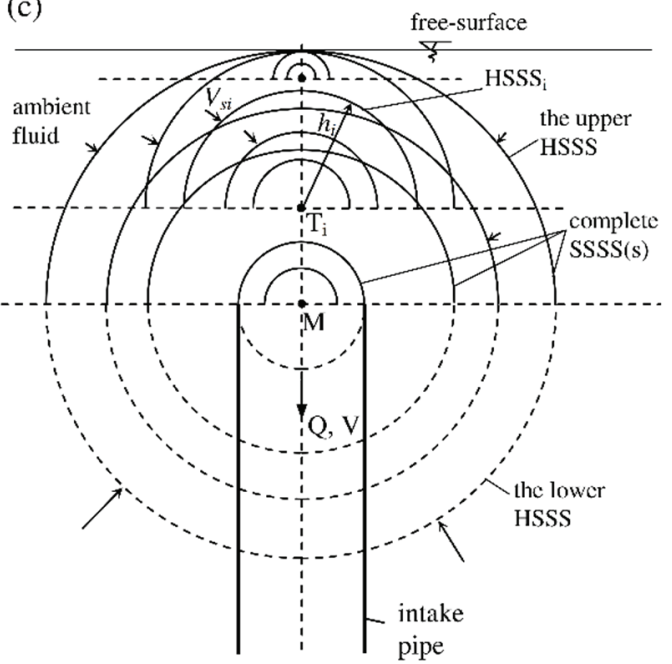

Figure 5 - HSSSs at different levels along the swirling vortex flow filament in case of a vertically flowing downward intake 
In the above analysis a vertically downward perfect straight vortex flow-filament on which there exist no boundary blockages is considered [profiles of the SSDs are perfectly symmetric about their vertical axis due to which HSSSs are perfect and complete, there cannot be velocity vectors below the horizontal plane (on which $T_{i}$ takes place) pointing $T_{i}$ ].

In practice the position, orientation and configuration of the intake-entrance, flow and geometrical conditions may be different, and there may exist boundary blockages on the profile of the SSDs due to which HSSSs may not be complete or HSSSs may be in any forms of SSSSs [because, there can be velocity vectors below the horizontal plane (on which $\mathrm{T}_{\mathrm{i}}$ takes place) pointing $\left.\mathrm{T}_{\mathrm{i}}\right]$ as indicated for an inclined intake in Fig. 6.

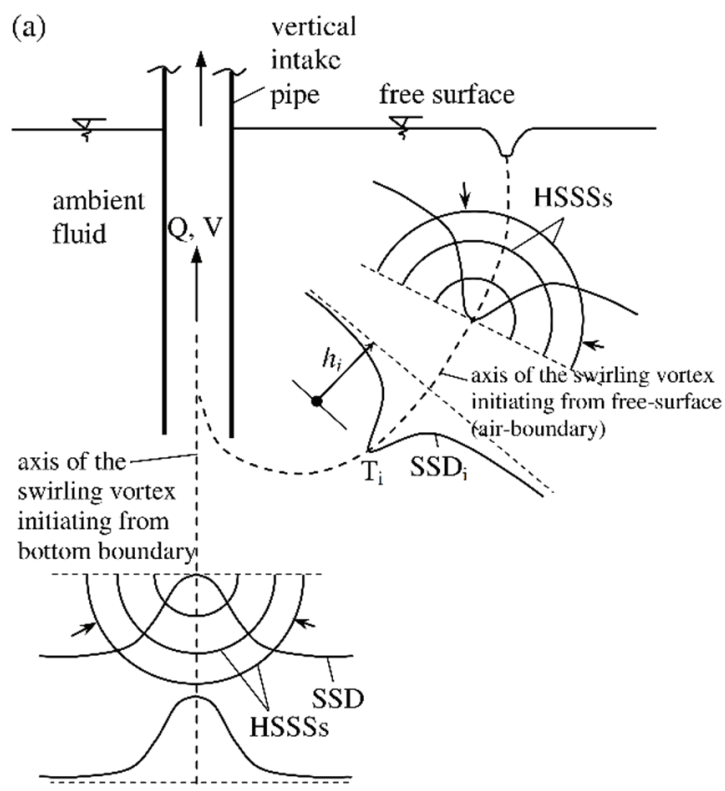

(b)

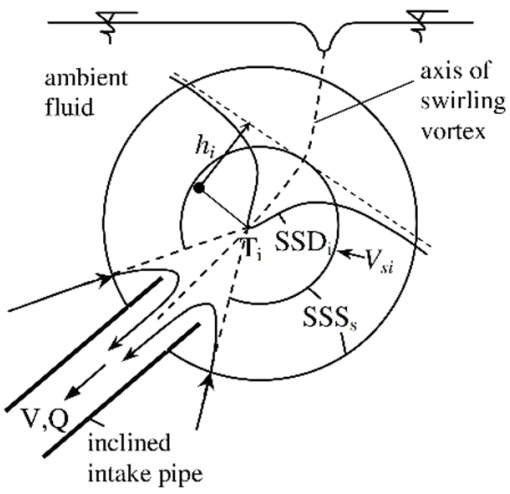

Figure 6 - HSSSs and SSDs along the swirling vortex flow filament in case of inclined intake(s) 
Basing on this fact, for general purposes it may be appropriate to rewrite Eqs. (12), and (13) as in Eqs. (14) and (15), respectively.

$$
\begin{aligned}
& A_{s i}=k \pi h_{i}^{2}=\mathrm{constant} \\
& q_{i}=A_{s i} V_{s i}
\end{aligned}
$$

where $A_{s i}=$ the effective surface area of the $\mathrm{i}^{\text {th }} \mathrm{SSSS}$ (or SSSS ); and $k=$ coefficient for the $\mathrm{i}^{\text {th }} \mathrm{SSSS}\left(\right.$ or $\mathrm{SSSS}_{\mathrm{i}}$ ) under consideration.

\section{VERIFICATION OF EXISTENCE OF HSSSs OR SSSSS}

The test data in the study by Suerich-Gulick et al. [15] relating to the constant velocity contours (isovels) on upstream side of a pipe intake issuing from a dead-end wall is replicated in Fig. 7.

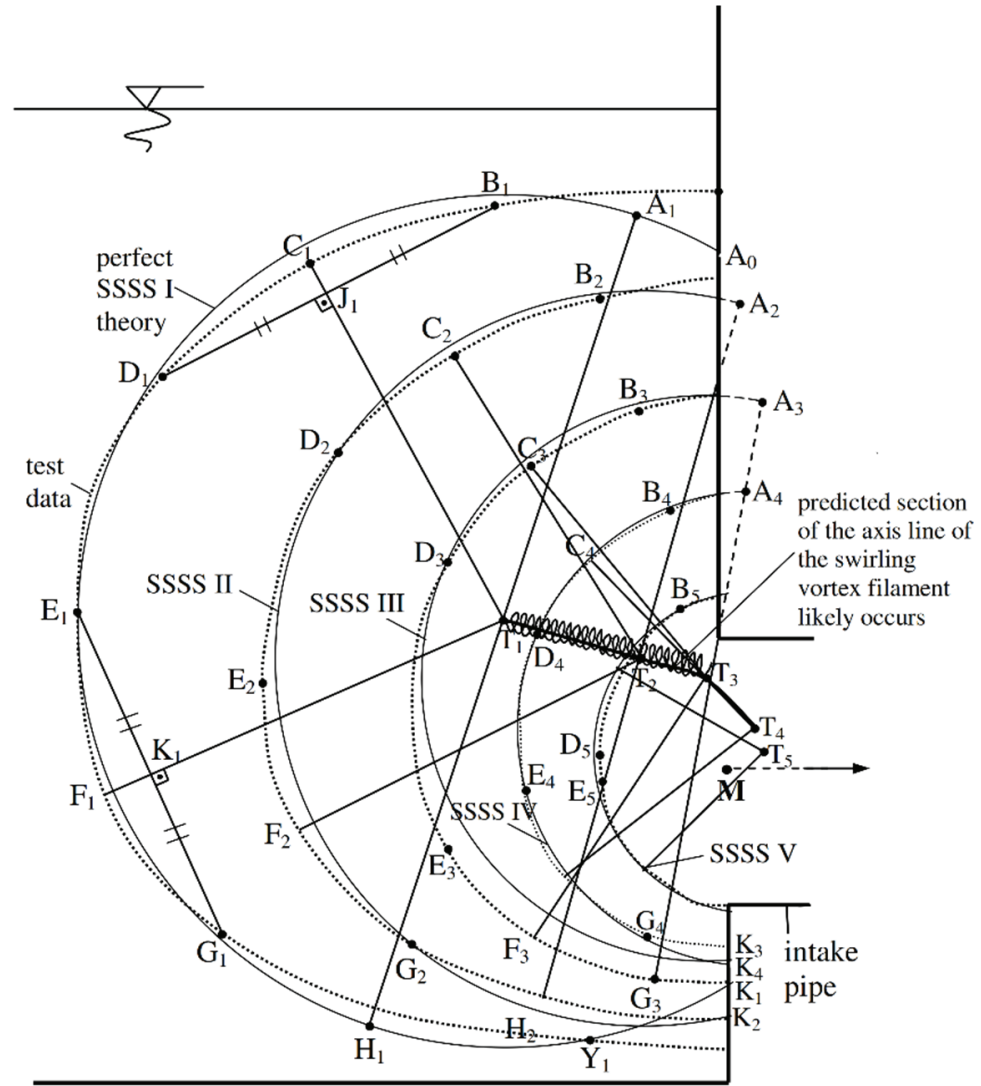

Figure 7 - Comparison of the theory and the available data in the study by Suerich-Gulick et al. [15] 
The clearances of the center of the intake to the dead-end wall and the bottom boundary are zero, and relatively small, respectively. In addition, there are two symmetric flow-disturbing vertical piers (with small clearance to the intake entrance) mounted on each side of the intakeentrance. Therefore, the velocity distribution in the ambient fluid region close to the intake should be under large boundary-effects that are clearly observable in the measured velocity contours in Fig. 7. It is a typical challenging case to test the method introduced in the present study. In Fig. 7 there are five isovels (HSSSs or SSSSs) that are denoted by SSSS I, II, III, IV, and V. By choosing arbitrarily chords (applied to the test data) and their locations the centers of SSSSs are geometrically located. For example, two arbitrarily chosen chords of $D_{1} J_{1} B_{1}$ and $E_{1} K_{1} G_{1}$ are used and thereby the center $T_{1}$ for SSSS $I$ is found as indicated in Fig. 7. By following the same geometrical procedure the centers $T_{2}, T_{3}, T_{4}$, and $T_{5}$ for SSSSs II, III, IV, and V, respectively are found. Not to make the figure crowded the chords for SSSSs II, III, IV, and V are not indicated in Fig. 7.

The line connecting the centers $T_{1}, T_{2}, T_{3}, T_{4}$, and $T_{5}$ (Fig. 7) is the approximate profile of the axis line of the swirling vortex flow-filament that is very similar to the one observed by several researchers (i.e., Quick [16], Figs. 2-7; Anwar et al. [2], Figs. 2-3). In practice the centers of HSSSs or SSSSs on downstream side of the intake-entrance center M are observed to be on the straight extension of the swirling vortex filament-line parallel to the axis of the intake pipe. But, Fig. 7 indicates that the predicted centers $T_{4}$ and $T_{5}$ are on downstream side of the intake center $\mathrm{M}$, and they are not exactly on the straight extension of the swirling vortex filament-line parallel to the inside-axis of the intake pipe. The reasons for this are as follows.

(1) The method may not give good results for the sections of SSDs whose centers remain within the intake since the approximations and assumptions under which the equations derived earlier (basing on those by Vatistas et al. [13]; Sun and Liu [8]) may not be acceptable in the fluid flow section within the intake pipe.

(2) The centers of the SSSs in Fig. 7 are found by applying geometry to the test data relating to the radial velocity on which boundary effects are obviously present and more pronounced in the flow region close to the boundaries (Fig. 7). Radial isovelocity contours deviate from being perfect spheres for the flow regions close to the solid boundaries (especially very confined intakes, i.e., intakes completely surrounded by very close solid boundaries).

(3) Arbitrarily chosen chords (applied to the test data-set) and their locations to predict the centers of SSSSs.

Figure 7 indicates that the agreement between method introduced in present study and the test data is satisfactory. The difference between the test data and the method is more pronounced in the flow regions close to the flow boundaries that is attributable to the effects of the boundaries as expected. In addition to visual comparison, to quantify the error between suggested method and the theory, the values of SSSS I, which has the maximum deviation with respect to the experimental data among the other SSSSs in Fig. 7, are used. The mean error is found as $4.2 \%$. Maximum error, which is obtained at close to the solid boundaries is about $12 \%$. Similar analysis can be done for other SSSSs in Fig. 7.

Figures 5-7 indicate that if an SSSS or HSSS cuts whole flow boundaries and thereby the SSSS along with boundaries completely encloses entire intake-entrance, $q_{i}$ through the net 
effective surface of the SSSS is the same as the intake discharge $Q\left(q_{i}=Q\right)$. Otherwise, $q_{i<}$ $Q$.

\section{CONCLUSIONS}

From this study, the following conclusions may be drawn.

- The discharge towards any point on the axis of the swirling vortex flow-filament from the free-surface to the intake can be calculated if the radial velocity at a given radius is measured.

- The flow towards the SSDs relating to the swirling vortex-filament has considerable effects on the radial velocity distribution within the ambient fluid flow region near the intake-entrance.

- The swirling vortex flow-filament consists of infinite number of coaxial SSSSs at every point along the axis of the swirling vortex flow-filament. Swirling vortex flow-filament is the result of the concurrently shrinking of the SSSSs along the axis of the swirling vortex.

\section{Symbols}

$\boldsymbol{A}_{\boldsymbol{s} i}:$ the net (effective) surface area of the $\mathrm{i}^{\text {th }} \mathrm{SSSS}\left[\mathrm{m}^{2}\right]$

$a$ : distance between the tip of the air-core vortex and center of the SSSS [m]

$\boldsymbol{H}_{\boldsymbol{r}}$ : water surface elevation at $R[\mathrm{~m}]$

$\boldsymbol{H}_{\mathbf{0}}$ : elevation of the lower end or tip of the air-core vortex [m]

$\boldsymbol{h} \quad$ : total depth of the air core [m]

$\boldsymbol{k} \quad$ : coefficient for the $\mathrm{i}^{\text {th }} \mathrm{SSSS}$ (or $\mathrm{SSSS}_{\mathrm{i}}$ ) under consideration

$\boldsymbol{q}_{i}:$ discharge towards the centre point $\mathrm{T}\left[\mathrm{m}^{3} / \mathrm{s}\right]$

$\boldsymbol{Q}$ : intake discharge $\left[\mathrm{m}^{3} / \mathrm{s}\right]$

$\boldsymbol{R} \quad$ : dimensionless radius

$\boldsymbol{R}_{\boldsymbol{s}^{*}} \quad$ : dimensionless radius of the $\mathrm{SSSS} *$

$\boldsymbol{r} \quad$ : horizontal radial distance from the vertical centerline of the vortex or intake entrance $[\mathrm{m}]$

$\boldsymbol{r}_{\boldsymbol{m}}$ : vortex core radius (outer limit radius of the viscous region) [m]

$\boldsymbol{S} \quad$ : submergence [m]

$\boldsymbol{S}_{\boldsymbol{c}} \quad$ : critical submergence $[\mathrm{m}]$

$\boldsymbol{V}$ : average intake-entrance velocity $[\mathrm{m} / \mathrm{s}]$

$V_{s i}:$ radial (normal) velocity at the $\mathrm{SSSS}_{\mathrm{i}}[\mathrm{m} / \mathrm{s}]$

$\boldsymbol{\beta} \quad$ : a coefficient

$\boldsymbol{\beta}_{\boldsymbol{i}} \quad$ : magnitude of $\beta$ for $\mathrm{SSD}_{\mathrm{i}}$ 


\section{References}

[1] Denny, D.F., An experimental study of air-entraining vortices in pump sumps. Proceedings of Institution of Mechanical Engineering, 170(2), 106-116, 1956.

[2] Anwar, H.O., Weller, J.A., Amphlett, M.B., Similarity of free vortex at horizontal intake. J. Hydraul. Res. 16(2), 95-106, 1978.

[3] Hecker, G.E., Fundamentals of vortex intake flow. In: Knauss, J (ed) Swirling Flow Problems at Intakes, pp. 13-38. A.A. Balkema, Rotterdam, 1987.

[4] Taştan, K., Yıldırım, N., Effects of Froude, Reynolds and Weber numbers on an airentraining vortex. J. Hydraul. Res. 52(3), 421-425, 2014.

[5] Kocabaş, F., Yıldırım, N., Effect of circulation on critical submergence of an intake pipe. J. Hydraul. Res. 40(6), 741-752, 2002.

[6] Yang, J., Liu, T., Bottacin-Busolin, A, Lin C., Effects of intake-entrance profiles on free-surface vortices. J. Hydraul. Res. 52(4), 523-531, 2014.

[7] Yıldırım, N., Eroğlu, N., Taştan, K., Occurrences of vortices at an intake of point sink character. Teknik Dergi, 23(1), 5799-5812, 2012.

[8] Sun, H., Liu, Y., Theoretical and experimental study on the vortex at hydraulic intakes. J. Hydraul. Res. 53(6), 787-796, 2015.

[9] Odgaard, A.J., Free-surface air-core vortex. J. Hydraul. Eng. 112(7), 610-620, 1986.

[10] Suerich-Gulick, F., Gaskin, S.J., Villeneuve, M., Parkinson, E., Characteristics of free surface vortices at low-head hydropower intakes. J. Hydraul. Eng. 140(3), 291-299, 2014.

[11] Vatistas, G.H., Lin, S., Li, P.M., A similar profile for the tangential velocity in vortex chambers. Exp. Fluids, 6 135-137, 1988.

[12] Anwar, H.O., Flow in a free vortex. Water Power, 4, 153-161, 1965.

[13] Vatistas, G.H., Kozel, V., Mih, W.C., A simpler model for concentrated vortices. Exp. Fluids, 11(1), 73-76, 1991.

[14] Taştan, K., Yıldırım, N., Effects of intake geometry on the occurrence of a free-surface vortex. J. Hydraul. Eng. 144(4), 04018009-1-04018009-11, 2018.

[15] Suerich-Gulick, F., Gaskin, S J., Villeneuve, M., Parkinson, E., Free surface intake vortices: Theoretical model and measurements J. Hydraul. Res. 52(4), 502-512, 2014.

[16] Quick, M.C., Efficiency of air-entraining vortex formation of water intake. Journal of the Hydraulics Division, 96(7), 1403-1415, 1970. 
